TableaU III

\begin{tabular}{|c|c|c|c|c|c|c|c|c|c|c|}
\hline $\begin{array}{c}\text { Nos } \\
1\end{array}$ & $m$ & $\begin{array}{c}m \omega \\
\mathrm{cm}^{2} \\
3\end{array}$ & $\mid \begin{array}{c}625+m \omega \\
k\end{array}$ & $\begin{array}{c}\text { ÉCARTEMENT } \\
\text { des armatures } \\
\text { transversales } \\
5\end{array}$ & $m^{3}$ & $\frac{\dot{V}}{V}$ & $\begin{array}{c}R b=50 \\
1+m^{\prime} \frac{V}{V} \\
8\end{array}$ & $\begin{array}{c}N \\
\mathrm{~kg} \\
9\end{array}$ & $\begin{array}{c}\frac{N}{625} \\
10\end{array}$ & $\begin{array}{c}\text { ComfFicrent } \\
\text { de sécurité } \\
\text { effectif } \\
11\end{array}$ \\
\hline $\begin{array}{l}1 \\
2 \\
3 \\
4 \\
5\end{array}$ & $\begin{array}{r}10 \\
12 \\
15 \\
9 \\
8\end{array}$ & $\begin{array}{r}71 \\
85 \\
106 \\
113 \\
226\end{array}$ & $\begin{array}{l}696 \\
710 \\
731 \\
738 \\
851\end{array}$ & $\begin{array}{l}0 \mathrm{~m} 20 \\
0,125 \\
0,0625 \\
0,25 \\
0,25\end{array}$ & $\begin{array}{r}8 \\
12 \\
15 \\
8 \\
8\end{array}$ & $\begin{array}{l}0,00401 \\
0,00802 \\
0,01604 \\
0,00404 \\
0,00400\end{array}$ & $\begin{array}{l}51,6 \\
54,8 \\
62,0 \\
51,6 \\
51,6\end{array}$ & $\begin{array}{r}35,913 \\
38,908 \\
55,322 \\
+18,080 \\
43,911\end{array}$ & $\begin{array}{l}57,4 \\
62,3 \\
72,5 \\
60,9 \\
70,2\end{array}$ & $\begin{array}{l}2,9 \\
2,8 \\
2,8 \\
2,8 \\
2,7\end{array}$ \\
\hline
\end{tabular}

de celle 4 du tableau I, donne les charges de rupture effectives correspondantes. En divisant les chiffres de la colonne 6 par ceux correspondants de la colonne 5 , on aura, dans chaque cas, le coefficient de sécurité effectif. On voit qu'il a des variations très considérables. Il varie entre 5 et 3,2 , ce qui indique que la formule [A], c'est-à-dire l'hypothèse de la constance de $m$, peut conduire à de sérieux mécomptes.

Faisant à présent les mêmes calculs en supposant $m$ variable, et suivant les règles indiquées dans la circulaire, on est amené, par des interpolations, à donner à $m$ les valeurs du tableau III.

D'autre part, on admet pour le béton, en nombre rond, d'après l'article 4 des instructions, une fatigue de 50 kilog., au lieu de celle de 35 admise ci-dessus, et en vertu de l'article 5, on majore cette fatigue d'après les coefficients de majoration:

$$
1+m \cdot \frac{V}{V}
$$

ce qui porte à :

$$
R_{\mathrm{b}}=50\left(1+m^{\prime} \frac{V^{\prime}}{V}\right)
$$

D'après les règles indiquées dans la circulaire, on est amené à prendre pour $m$ les valeurs du tableau III. Les charges $N$ à faire supporter à la colonne seront données par la formule:

$$
N=\mathrm{R}_{\mathrm{b}}(625+m \omega)
$$

On a ainsi le tableau III.

Les chiffres de la colonne 9 sont obtenus par la formule [B']. Ceux de la colonne $\mathrm{I}$, en divisant la valeur des charges de rupture (tableau I, colonne 4) par les chiffres de la colonne ro. Et, ici, on voit que les coefficients de sécurité effectifs ont une constance remarquable; ce qui permet d'être plus hardi sur la fatigue théorique maxima à admettre.

III et IV. - Exécution des travaux et épreuves des ouyrages. - Les instrucions sur ces deux matières se justifient d'elles-mêmes, et il n'y a pas à s'y arrêter ici.

En résumé, la Commission a fait son possible pour donner aux ingénieurs des instructions aussi précises que le comporte le sujet, à éclaircir ces instructions en tant que de besoin par la circulaire à y joindre, et à faciliter les calculs de résistance à ceux des ingénieurs qui le désirent, le tout sans empiéter en rien sur leur libre arbitre, lequel doit rester, ici, plus absolu que partout ailleurs, puisqu'il s'agit d'une province nouvelle dans l'art de bâtir, qui s'offre à leurs études et à leur activité, et dans laquelle, d'ailleurs, plusieurs d'entre eux ont été parmi les premiers pionniers qui ont préparé les voles actuellement suivies.

L'Inspecteur général, Président et rapporteur, de la Commission,

(A suivre.) Maurice LEvy.

\section{Le Développement de la Traction électrique ${ }^{(*)}$}

Le succès obtenu par les tramways électriques fit songer à des applications plus étendues de ce système de traction. D'une part, le besoin sans cesse croissant de moyens de transport dans les villes rendait nécessaire la construction où l'extension des chemins de fer métropolitains, aé. riens ou souterrains, pour lesquels la traction électrique était tout indiquée; d'autre part, on songeait à établir de nouvelles jonctions interurbaines qui, pour certains réseaux particuliers, se prètaient mal à l'exploilalion par locomotives á vapeur; enfin, on sentait la nécessité d'électrifier certaines portions de quelques grandes lignes do chemins de fér établies én tunnel ou en soulerrain.

Parmi les avantages les plus importants qu'offre la traction électrique, il faut citer la possibilité d'utiliser pour la propulsion des trains l'énergie des chutes d'eau, et de n'ê. tre plus tributaire des mines de charbon de l'étranger. Cet avantage avait été nettement senti par le gouvernement italien qui, dès 1900, entrait résolument dans la voie de l'électrification des chemins de fer, et commençait par écuiper, dans le nord de l'Italie, deux lignes de plus de 100 kilomètres de longueur. L'une, reliant Milan à Gallarate et Varèze, sur le lac Majeur, était équipée à courant continu avec prise de courant par troisième rail. L'autre, celle de Lecco à Sandrio et Chiavenna, par la vallée de la Valtelline, fonctionne avec du courant alternatif triphasé à 3000 volts, 16 périodes.

En 1902-1903, l'Allgemeine Elettricitäts Gesellschaft procédait aux célèbres expériences de Zossen, qui ont été décrites dans La Houille Blanche de janvier 1904. Enfin, á l'heure actuelle, le nombre des lignes à traction électrique à long parcours, et à grand trafic, devient de plus en plus élevé.

La traction électrique présente, sur la traction à vapeur, certains avantages importants. Ces avantages se manifestent par un accroissement considérable de trafic sur les lignes électrifiées. On peut citer de nombreux exemples de cel accroissement, et, d'après M. de Valbreuse, sur le chemin de fer de Manhattam à New-York, le trafic a augmenté de 50 pour 100 dans la première année, alors que les frais d'exploitalion tombaient de 55,79 à 40,20 pour 100 des recettes brutes. Sur la ligne Milan-Gallarate-Varèze, l'accroissement de trafic a été de 170 pour 100 en trois ans. Ce résul. tat provient surtout de ce que l'emploi de la traction électrique permet une méthode rationnelle d'exploitation, avec des convois fréquents et relativement légers; les trains sont

(4) Résumé d'une conférence faite le 9 janvier 1907 à la société Internationale des Electriciens par M, de VaLbreuse, et de la discussion qui s'en est suivie, lors des séances des 6. février et 10 avril. 
plus rapprochés, plus rapides et plus confortables qu'avec la traction à vapeur.

Au point de vue des dépenses de charbons, qui d'ailleurs ne constituent tout au plus en général que 20 pour 100 des dépenses totales d'exploitation d'une compagnie de chemins de fer, il est facile de voir que, malgré les multiples transformations de l'énergie, on arrive à peu près aux mômes chiffres avec la traction électrique qu'avec la vapeur; en effet, le rendement total des locomotives est certainement inférieur à celui des machines fixes; d'autre part, la mise en pression, ou le maintien des feux sur les locomotives, consomme, en pure perte, une quantité importante de combustible. En outre, on peut réunir dans les stations centrales des unités génératrices très puissantes, ayant un rendement unitaire très élevé. Enfin, les conditions sont évidemment tout à fait favorables à la traction électrique, lorsqu'on dispose à bon compte d'énergie hydraulique sous forme de chutes d'eau.

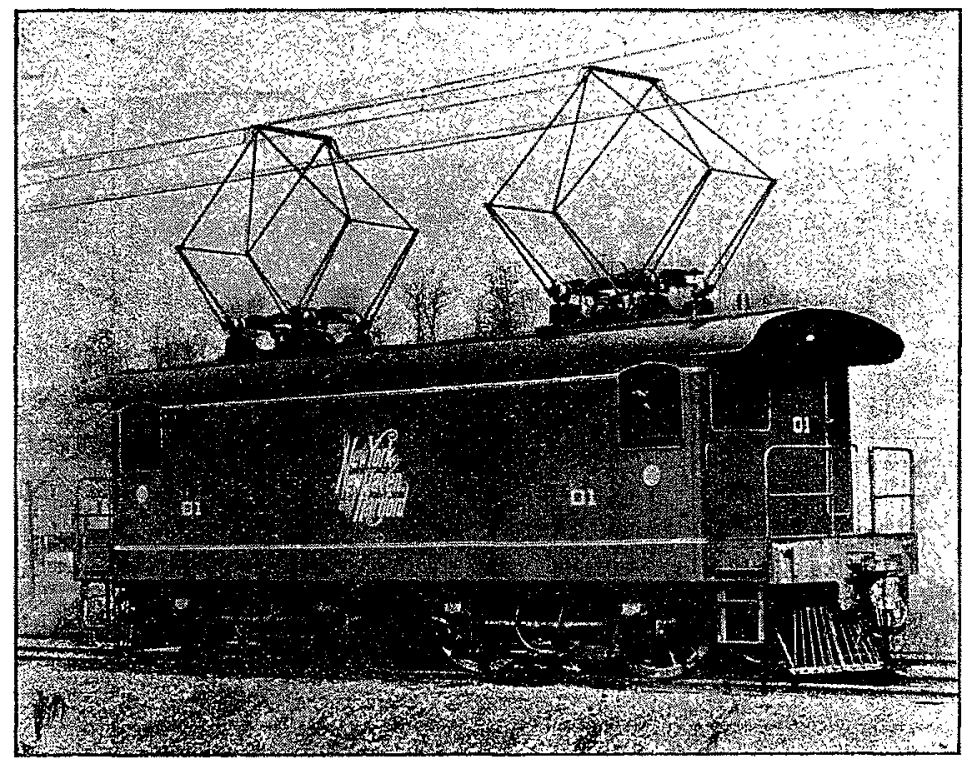

Fig. 1. - Locomotive du New-York, New-Haven et Hartford.

Au point de vue de la vitesse, il ne faut pas s'attendre à voir réaliser avec la traction électrique des vitesses maxima bien supérieure à celles qu'atteignent les locomotives á vapeur ; mais, par suite de la valeur très élevée de l'accélération que l'on peut obtenir avec les trains électriques, les démarrages sont très rapides, et la vitesse moyenne diffère peu de la vitesse maxima. Pour les trains de banlieue, à arrêts fréquents, il y a là un avantage marqué pour la traction électrique, dont la vitesse commerciale devient supérieure à celle qu'on obtient avec la traction à vapeur.

Signalons encore, à l'avantage de la traction électrique, l'absence de toute escarbille incandescente, susceptible de mettre le feu aux herbes, aux récoltes ou au bois environnants, ainsi que cela n'arrive que trop souvent en temps de séchuresse avec les locomolives à vapeur. Aux Etats: Unis, les compagnies de chemins de fer sont obligées de payer de très grosses primes d'assurances contre ces risques d'incendie.

Trois système principaux sont employés pour la traction èlectrique: le courant continu, à basse ou moyenne tension; le courant triphasé, et enfin le courant alternatif monophasé.

Comparaison des divers systèmes de traction électrique. - Les frais d'établissement de l'usine centrale génératrice sont à peu près les mèmes pour les différents sys- tèmes de traction; toutefols, une usine avec alternateurs monophasés coute plus cher qu'une usine à courants triphasés.

Le courant continu à basse tension (600 volts) a l'avantage d'employer un matériel roulant relativement peu couteux. En outre, les moteurs à courant continu présentent un rendement supérieur à celui des autres moteurs; ils sont plus économiques au point de vue des pertes de charge et sont moins encombrants ( $\left.{ }^{*}\right)$. Mais par, contre, la preduction de l'énergie électrique sous forme de courant alter. natif dans une station centrale; et la conversion de ce courant alternatif en courant continu dans des sous-stations de transformation alimentant la ligne, condưisent à des frais d'installation et d'exploitation extrêmement élevés.

Aver le système triphasé; les frais d'installation de la ligne sont beaucoup moins importants qu'avec le continu, la surveillance et l'entretien de celle-ci ne conduisent qu'à de faibles dépensés: D'autre part, léquipement du matériel roulant n'est pas très coutenx. Les moteurs triphasés sont des machines des plus robustes, n'ayant pas de collecteur, et d'un entretien minime.Avec de faibles entrefers,

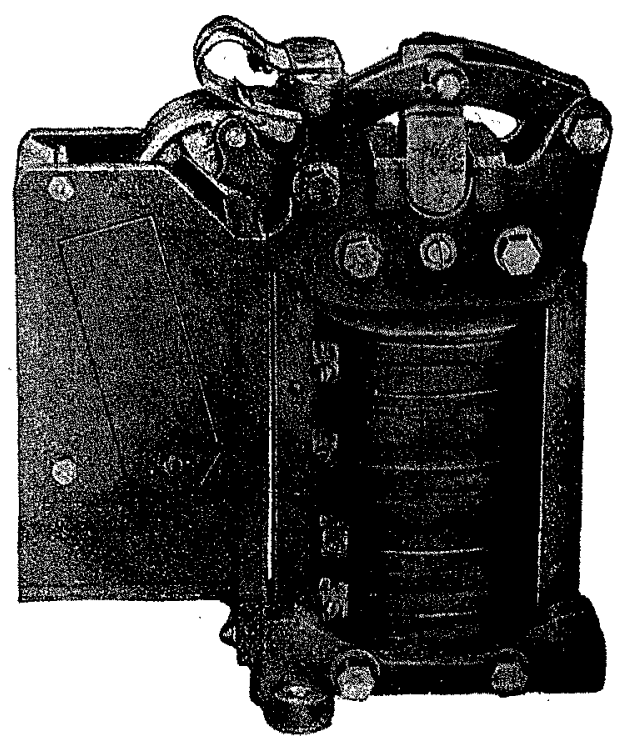

Fia. 2. - Contacteur a courant alternatif. système Thomson-Houston. leur rendement est bon, sauf pour le groupement en cascade.

Le rendement global de l'ensemble, ligne et moteurs, est supérieur à celui du système continu à 600 volts. Le ren: dement total se trouve encore amélioré par la récupération aux descentes dans les pays de montagnes.

Avec le système monophasé, les frais d'installation et d'entretien de la ligne sont encore plus faible qu'avec le triphasé. Par contre, l'équipement et l'entretien du matériel roulant est plus éle vé que dans le cas du courant continu. Au total, il semble cependant que les frais d'équipement de la ligne et du matériel roulant réunis soient inférieurs de 10 à 15 pour 100 à ceux du système à courant continu.

Avec le systeme à coúrant continu à moyenne tension, il semble que les frais totaux soient inférieures à ceux du systèmé monophasé, s'il s'agit d'une ligne dontla longueur' ne dépasse pas une une trentaine de kilometres au maximum.

Quand les arrêts doivent êtres fréquents, il faut un grand couple de démarrage pour obtenir une bonne vitesse moyenne. Comme le couple de démarrago des moteurs série á courant continu est le plus élevé, on est conduit dans cé cas à employer le courant continu. Pour que l'exploitation soit rénumératrice, il faut que le traffc soit intense. Si ce n'est pas le cas, on est conduit a adopter le système monophasé.

(") A propos de la comparaison des systemes à courant continu et à cotrants alternatifs, voir la commication sur ce sujet faite par $M$. Auvent, au 35' Congrès de l'Associalion prancaise pour l'Avancemen des Sciences, tenu à Lyon en 1906 (La Houille Blanche, Aout 1006). 
Pour un service de montagne, où la récupération joue un rôle important, et où généralement il n'y a pas un trafic considérable, on aura avantage à recourir au système triphasé. Pour la remorque des trains lourds, sur une ligne de trafic restreint, le système triphasé pourra souvent lutter avec avantage contre le système monophasé. Il permet en effet l'emploi de moteurs extrêmement robustes, de grande puissance, ne nécessitant que très peu d'entretien, et il présente Jans son ensemble un très bon rendement.

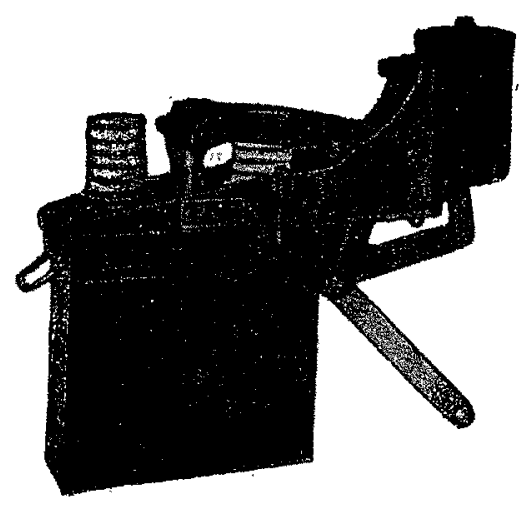

Fra. 3. - interrupteun à huile Thomson Houston.

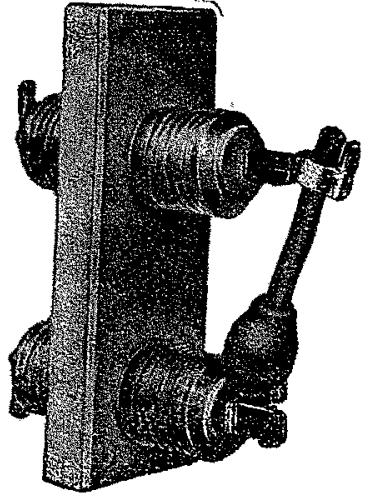

FlG. 4. - Fusible à expulsion pour 6600 volts.
Quant au système monophasé, il s'applique admirablement à toutes les lignes où le trafic n'est pâs très intense, et oú les frais d'installations et d'exploitation doivent être bas. Télles sont les jonctions interurbaines, les chemins de for secondaires el d'intérêts locaux. Son emploi peut rendre d'immenses services, et transformer les conditions d'existence dans un grand nombre de contrées, en créant des moyens de communication commodes, fréquents et rapides. Il a cela d'excellent, c'est que l'on peut faire varier la tension aux bornes des moteurs (et par cela même la vilesse) par simple changement de bornes du secondaire des transformateurs, sans avoir à perdre de l'énergie dans des résistances, comme cela a lieu pour les autres systèmés. Il présente aussi l'avantage considérable de permettre le račcordement de ses lignes aux réseaux urbains.

Traction par courant continu. - Sur la ligne MilanGallarate-Varèze, qui est longue de 130 kilomètres, l'intervalle entre deux sous-stations voisines est d'une quinzaine de kilomètres; c'est là un maximum, en général cette distance est réduite a 5 ou 6 kilométres.

Parmi les locomotives à courant continu, on peut signaler celles du New-York Central and Hudson River Railroad, ligne à trafic très intense qui, depuis 1905 , est équipée sur 90 kilométres environ avec le sytème à courant continu à 600 volts. Les locomotives sont portées par six essieux, dont les 4 du milieu sont moteurs. Chaque essieu moteur porte, directement calé sur lui, l'induit d'un moteur. série bipolaire, les inducteurs des 4 moteurs sont placés horizontalement, en série les uns derrière les autres, et forment un système magnétique complet, constitué par une carcasse unique (*).

Dans les nouvelles installations, on tend de plus en plus à se servir de la traction à unités multiples, qui permet de répartir les moteurs sur la plupart des voitures qui composent les trains. Dans ce cas, le contrôleur bien connu

(*) Pour plus de détails sur cette locomotive, se reporter à la description qui en a été faite dans La Houllle Blanche de mars 1905. des voitures ordinaires de tramway est remplacé par une série d'interrupteur's spéciaux à contact, appelés contacteurs, qui sont manœuvrés par des dispositifs électropneumatiques oli électromagnétiques. Dans le premier cas (système employé par la Compagnie Westinghouse), les contacteurs sont entrainés par les pistons de cylindrés à air comprimé, dont les valves sont manœuvrés par de petits solénoïdes; ceux-ci sont excités, à la volonté du mécanicien, par un courant local fourni par une petite batterie d'accumulateurs. Dans le second cas (système de la Compagnie Thomson-Houston, de l'A. E. G., etc.), la commande des contacteurs est effectuée par des électroaimants qu'excite, à la volonté du mécanicien, un courant local dérivé du circuit principal. Dans l'un et l'autre cas, le rôle du mécanicien se borne à manœuvrer un pelit contrôleur, ou manipulateur, établi pour de très faibles intensités, et commandant les divers contacteurs du train. Tout ceci s'applique aussi bien au courant alternatif qu'au courant continu.

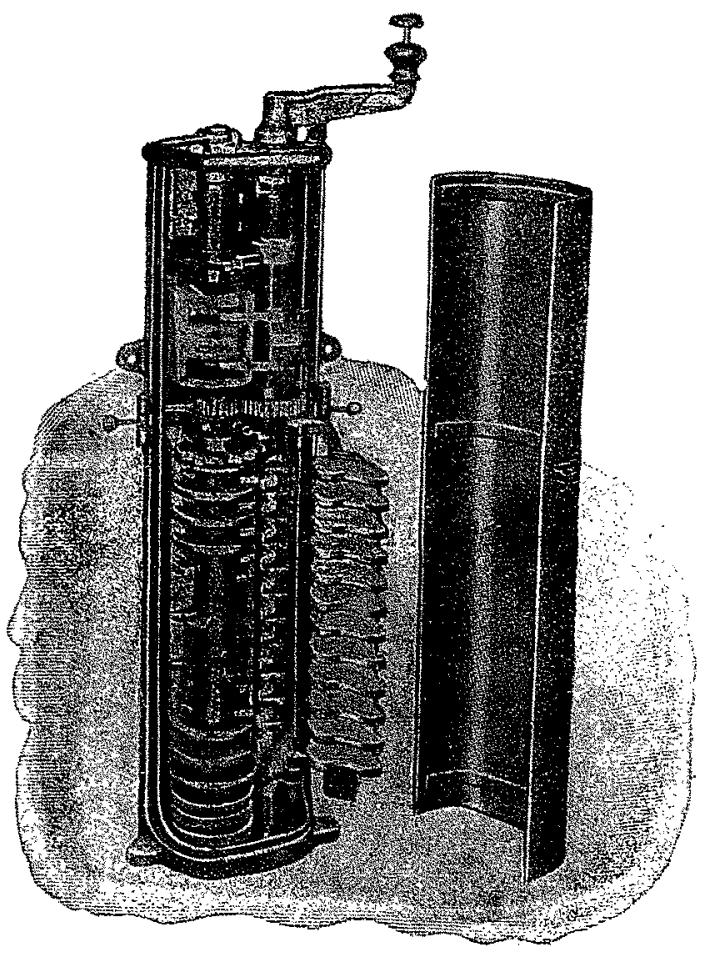

Fig. 5. - Contrôleur de commande Thomson-Houston pour équipement à unites multiples.

Ce dispositif est généralement complété par un limiteur d'intensité, appareil qui empeche automatiquement le passage du système à une vitesse supérieure, lorsque lintensité absorbée par les moteurs excède une valeur déterminée.

Ce disposilif s'applique également aux locomotives puissantes, pour lesquelles l'intensité du courant atteint une valeur considerable au démarrage, telle que l'on ne pourrait songer à faire passer tout le courant par un seul contrôleur ordinaire.

La question de l'emploi de batteries-tampons d'accumulateurs dans les sous-stations est assez controversée. Si l'on emploie une batterie-tampon, il suffit que la puissance des machines installées soit égale à la puissance moyenné que la sous-station est appelée à fournir; les machines travaillent alors à peu près toujours à pleine charge. Si au contraire l'on n'emploie pas de batterie-tampon, il faut que les machines installées, aussi bien à la sous-station qu'à l'usine génératrice, présentent une puissance supé- 
rieure à la puissance moyenne; les feeders doivent euxmêmes être prévus plus largement. Par contre, cette solution permet d'éviter les frais considérables d'amortissement et d'entretien d'une batterie.

Notons toutefois qu'on est unanime a reconnaitre que la présence d'une batterie suffisamment puissante dans une sous-station donne une plus grande sécurité en permettant de continuer à assurer le service en cas d'un arrêt mómentané, n'excédant pas 20 à 30 minutes, des machines génératrices ou transformatrices, ou bien encore d'une interruption de courte durée des lignes primaires.

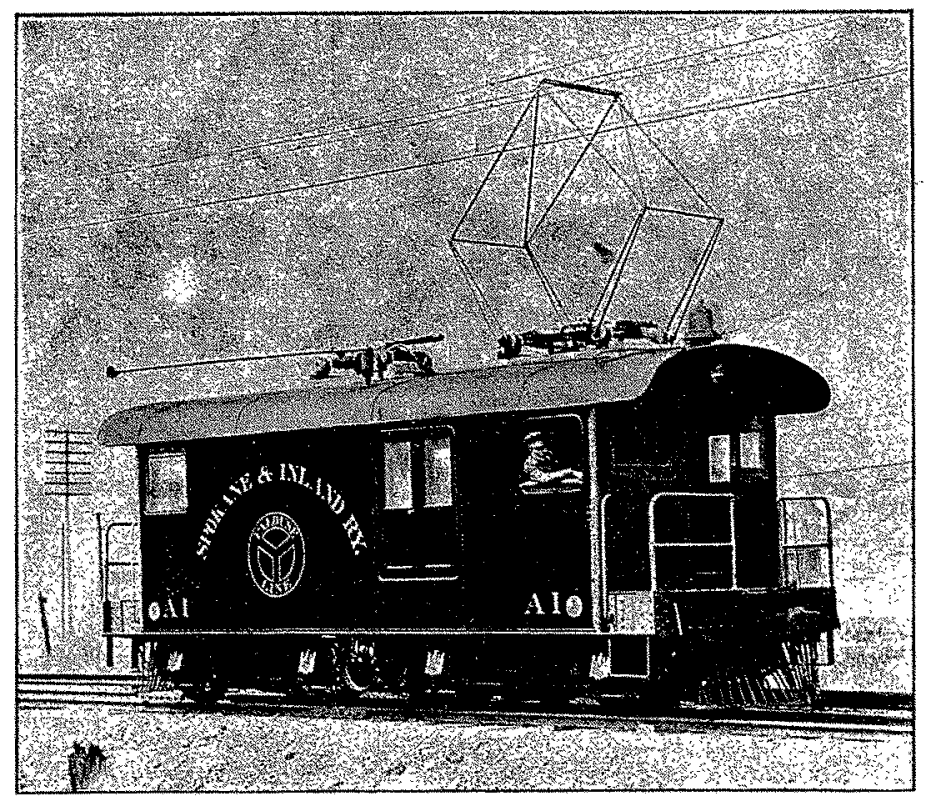

Fra. 6. - Locomotive de la ligne de Spokane.

Su' la ligne Milan-Gallarate-Varèze, l'équipement primitif ne comportait pas de batteries d'accumulateurs; on s'est vu obligé d'en ajouter depuis. En Angleterre, il y a une tendance marquée à la suppression des batteries, el, à l'heure actuelle, aucune sous-station des différents réseaux métropolitains électriques n'en est pourvue. En France, la ligne des Invalides à Versailles a toujours parfaitement fonctionné sans batterie, bien que les fluctuations de charge y soient très importantes. Par contre, le Métropolitain emploie de puissantes batteries pour uniformiser la charge des machines.

$\mathrm{Au}$ Métropolitain souterrain de New-York, on n'a pas installé d'accumulateurs; on y aura recours plus tard si le besoin s'en fait sentir. Cependant, en Amérique, on apprécie généralement les services que rendent les batteries-tampons. Dans les installations du New-York-Central Railway, chacune des huit sous-stations contiendra une batterie d'accumulateurs de 300 à 4000 ampères-heure, au régime de décharge en une heure, ce qui leur permettrait d'assurer, à elles seules, le service de la ligne pendant une heure.

Lorsque la longueur de la ligne est rélativement peu considérable, tout en étant cependant trop grande pour l'emploi économique du courant continu, il peut être avantageux de recourir au système à courant continu. à moyenne tension.

En 1903, un premier essal de traction par ce systeme a été fait par M. Thury, sur la ligne du chemin de fer de Saint-Georges-de-Commiers à La Mure, avec une distribution du courant continu a trois ponts, et avec une difference de potentiel de 1200 volts entre chaque pont. Ces essais ont donné des rósultats très satisfaisants $\left(^{*}\right)$.

En 1906, on a ouvert a l'exploitation la ligne de Bonn à Cologne, équipée par la Société Siemens-Schuckert avec du courant continu à 1000 volts. Chaque automatrice est actionnée par deux moteurs tétrapolaires de $130 \mathrm{HP}$, munis de pôles de commutation. Les projets fails, d'une part avec le courant monophase à 6000 volts, et d'autre part avec le continu à 1000 volts, ont montré que le prix de l'équipement électrique des voitures motrices serait 1,7 fois plus élevé dans le premier cas que dans le second, et que le poids de cet équipement serait à peu près le double, entrainant ainsi une augmentation corrélative de l'ènergie électrique dépensée. L'installation électrique est complétée par trois batteries d'accumulateurs, dont une est à l'usine génératrice, et les deux autres en cours de route.

La Société Siemens-Schuckert vient de mettre en service une ligne de 14,5 kilomètres pour le transport des minerais entre Maizières et Sainte-Marie-aux-Chênes., Cetle ligne est alimentée par du courant continu à 2000 volts. Chaque train pèse 320 tonnes, et est remorqué par une locomotive de 56 tonnes, munie de 4 moteurs de 160 chevaux.

La ligne de Bellinzona à Mesono, ouverte a l'exploitation dans le courant de l'automne passé, est alimentée par du courant continu à 1600 volts. Chaque automotrice porte 4 moteurs de 75 chevaux.

Signalons enfin les essais falts par la Société Krizik, en vue de l'électrification du métropolitain de vienne, dont la longueur est de 27,6 kilométres. La tension d'alimentation

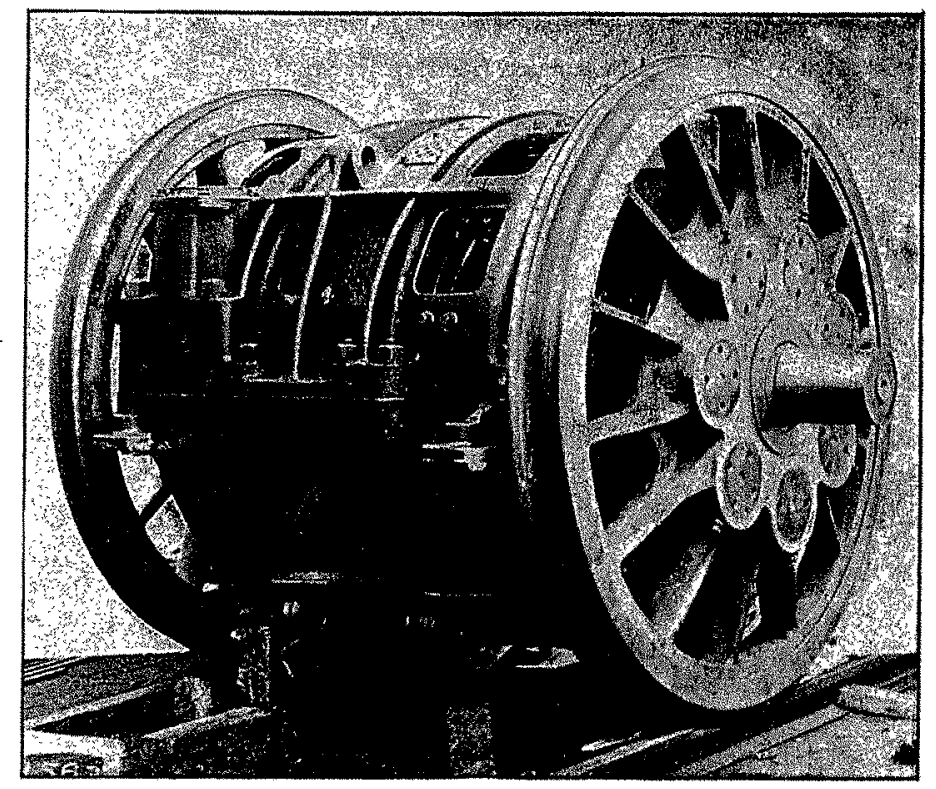

Fla. 7. - Vue d'un essieu de la locomotive du New-York, New-Haveń et Hartford, avec son moteur.

est de 3000 volts, et est répartie sur deux ponts de 1500 volts, constitués par deux fils aériens distants de $1 \mathrm{~m} 40$ et par les rails de retour. Ce système avait déjá donné toute satisfaction à la société Krizik sur la ligne de Tabor à Béchyne, fonctionnant avec 1400 volts, entre flls extremes.

() La description de l'installation Glectrique du chemin de fer de Saint-Georges-de-Commiers à La Mure a été faite dans La Houllle Blanche de 1903. Pour les essais, voir aussi La Houllle Blanche de mars 1906 . 
Traction par courants triphasés. - En dehors des lignes de tramways à courant triphasé, ce mode de traction a été encore appliquée aux véritables lignes de chemins de fer de Ia Valteline par la maison Ganz en 1902, et du Simplon par la maison Brown-Boveri en 1906.

On trouvera la descriptien des installations électriques de la ligne de la Valteline dans La Houille Blanche d'octobre 1903. Au début, chaque train de voyageurs comprenait une automotrice et quelques voitures de remorque; les trains de marchandise élaient trainés par des locomotives. Dans la suite, on a construit des locomotives capables de remorquer indifféremment, soit les trains de voya. geurs, soit ceux de marchandises.

Ces locomotives comprennent trois essieux moteurs et deux porteurs exirêmes, chacun de ces deux derniers formant, avec l'essieu moteur voisin, un bogie susceptible de tourner autour d'un pivot. Deux moteurs de $450 \mathrm{HP}$, fixés au chassis, entrainent les trois essieux par l'intermédiaire de bielles et de barres d'accouplement.

Chaque moteur est double, la même carcasse contenant à la fois un moteur primaire et un moteur secondaire invariablement réliés l'un à l'autre; dans le moteur primaire, l'inducteur est fixe et l'induit mobile; dans le moteur secondaire c'est l'inducteur qui tourne et l'induit qui est fixe. En grande vitesse (64 kilomètres à l'heure), les moteurs primaires travaillent seuls; en petite vitesse (32 kilomètres) chaque moteur secondaire est accouplé en cascade avec l'un des moteurs primaires. Pour les vitesses de transition, on intercale des résistances variables dans le circuit du rotor du moteur primaire ou dans celui du moteur secondaire.

Les dernières locomotives livrées par la maison Ganz ne sont plus établies de la même façon. Un moteur à 8 pôles assure la marche à grande vitesse; sa puissance est de $1500 \mathrm{HP}$. Un second moteur à 12 pòles assure la marche en pelite vitesse, sa puissance est un peu moindre. Le groupement en cascade de ces deux moteurs permet de marcher à une vitesse d'environ 25 kilometres à l'heure.

La traction électrique dans le tunnel du.Simplon est faite avec du courant triphasé, ainsi qu'on l'a vu dans le précédent numéro de La Houille Blanche. Les prochaines locomotives que la maison Brown-Boveri mettra en servicesur la ligne du Simplon auront quatre essieux moteurs, utilisant pour l'adhérence le poids total de la machine. Ces locomotives auront quatre vitesses, obtenues par modification du nombre des poles du stator, et par groupement en étoile et en triangle, des enroulements du rotor,

L'administration des chemins de fer de l'Etat italien vient de confier à la Compagnie Westinghouse l'électrification de la partie de la ligne de Gênes à Turin qui est comprise entre Busalla à Pontedecimo, et dans laquelle se trouve le tunnel des Giovi, dont l'exploitation est particulièrement difficile.

Les détracteurs du systeme de traction par courant triphasé ont. signalé souvent que les fortes variations de charges, produites sur la ligne lorsqu'un train monte ou descend, doivent amener des varialions de tension importantes, nuisibles au. fonctionnement des moteurs des autres trains, dont le couple est proportionnel au carré de la tension. En réalité, comme l'a montré $M$. Boucherot, il se produit un tout autre phénomène : au moment d'unè forte surcharge due à un train, la vitesse des groupes générateurs de lusine diminue, la fréquence des courants baisse, et les moteurs des autres trains en marche se trouvant au synchronisme, ou même au-delà, n'absorbent plus de courant, ou font de la récupération. L'ensemble des trains forme donc un vaste volant, et, en fait, les variations maxima de charge constatées à l'usine génératrice de Morbegno, n'ont jamais dépassé 70 à 80 pour 100 . On pourrait même abaisser à une valeur insignifiante cette variation de charge, en admettant de plus grands écarts de réglage de la vitesse des groupes générateurs,

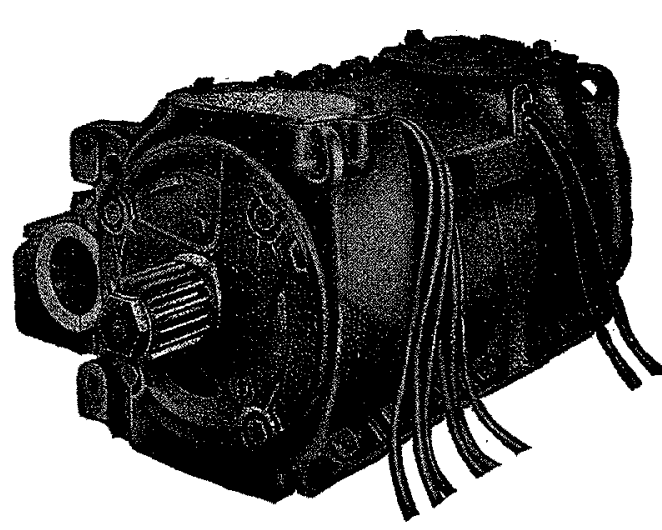

Fra. 8. - Moteur série compensé ThomsonHouston, fonctionnant en altermatif et en continu. et, par suite, dè plus grandes variations de la fréquence, de manière à accentuer l'effet précédent.

M. Gratzmuller fait remarquer que les moteurs polyphasés, à rotor en court-circuit,etsur. tout ceux en cage d'écureuil, présenteraient des conditions particulières de sécurité et d'encombrement réduit, si lon pouvait les démarrer et faire varier leur vitesse, quitte à introduire le collecteur à lames, mais, celte fois, à un endroit choisi à volonté.

On peut réaliser ce desideratum en alimentantles moteurs à fréquence et à voltages variables. Si, à l'arrêt, on fait tourner, en valeur absolue, le champ résultant à une vitesse voisine de sa vitesse relative rapportée au rotor en marche normale, on obtiendra un couple égal au couple normal; pour cela, il faudra évidemment alimenter le stator avec un courant d'une fréquence égale à $g \omega$, si $\omega$ est la fréquence du réseau et $g$ le glissement.

on peut facilement réaliser la variation du voltage et de la fréquence au moyen d'un collecteur à lames spécial, analogue à celui qui a été décrit dans $L a$ Houille Blanché d'avril 1905.

Terminons en signalant que le système triphasé ne peutpas se prêter à l'emploi d'unités multiples, car, par suite d'une faible inégalité de diamètre des roues des différentes voitures automotrices, due à l'usure des bandages, certains moteurs présenteraient un glissement anormal, tandis que d'autres tourneraient à peu près au synchronisme. Sur les locomotives triphasées, comme sur celles à vapeur, on pare à cet inconvénient en remplaçant simultanément les bandages de tous les essieux moteurs.

Traction par courant monophasé. - Depuis longtemps, on a établi des moteurs série de faible puissance, à inducteurs feuilletés, fonctionnant sur courant alternatif. Le sens du courant variant simultanément dans les bobines de l'inducteur et de l'induit, on obtient un couple pulsatoire toujours dể même sens; mais, à côté de l'action dynamique, due à la rotation de l'induit dans le champ magnétique, il se produit, comme dans un transformateur, une action statique, d'après laquelle le flux altematif, engendré dans l'inducteur agissant comme primaire, induit une force électromotrice alternative dans les circuits secondaires fermés, constitués par les bobines induites que court-circuitent les balais.-D'autre part, pour obtenir une valeur suffisamment élevée du facteur de puissance, on est obligé d'adopter un très faible entrefer, de solte que la réaction d'induit produit des effets intenses qui rendent la commutation franche- 
ment mauvaise dès que la puissance du moteur devient un peu importante.

On a donc été conduit d'une part à employer, sur l'inducteur, un enroulement compensateur transversal, ou des pôles de commulation destinés à neutraliser le flux propre de l'induit, et, d'autre part, à recourir à des artifices pour limiter la valeur de l'intensité des courants produits dans les bobines en court-circuit, par les forces électromotrices induites statiquement. Caśs artifices consistent, généralement, dans l'emploi de jonctions résistantes interposées entre les lames du collecteur et les points correspondants de l'induit. Dans certains moteurs, on emploie un enroulement auxilliaire produisant un champ déphasé sensiblement de $90^{\circ}$ par rapport au champ principal.

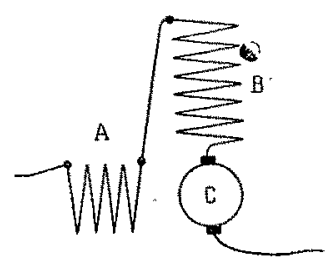

FIG. 9.

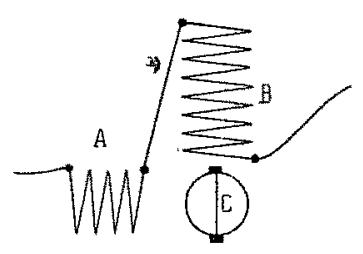

FIG. 10.

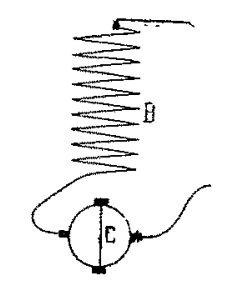

Fia. 11.
Le moteur série monophasé est schématiquement représenté par la figure 9. Malgré tous les artifices employés, on ne peut, on général, établir de pareils moteurs pour des différences de potentiel aux bornes supérieures a 250 volts, et pour des fréquences de plus de 25 périodes par seconde.

Un autre type de moteur est celui du moteur à répulsion d'Atkinson, représenté par la figure 10. Ce moteur fonctionne d'une façon très satisfaisante au synchronisme, car' il se forme alors un "flux tournant à la mème vitesse que l'induit. M. Marius Latour le considère comme un véritable alternateur, en ce sens que, bien qu'un enroulement d'excitation A soit disposé sur le stator, le rotor se comporte comme un inducteur à pôles fixes, et le stator comme un induit d'alternateur. Le moteur à répulsion est d'autant meilleur que la tréquence est plus élevée.

M. Marius Latour et MM. Winter et Echberg ont eu l'ingénieuse idée de combiner les deux moteurs précédents, en profitant à la fois du grand couple de démarrage du moteur série, et de la bonne commutation du second au voisinage du synchronisme ; ils ont ainsi réalisé le moteur à répulsion compensé, schématiquement représenté par la figure 11. Le couple est alors produit entre le champ du rotor $\mathrm{C}$ et les ampères tours de $\mathrm{B}$.

Le moteur à répulsion compensé est, d'après $M$. Latour, un alternateur monophasé auto-excitateur. L'excitation du rotor peut, avec un transformateur appropriè, être monté en shunt au lieu de l'être en série. Si la connexion shunt est établie en vitesse, on peut faire de la récupération sur les pentes, au mềme titre que les moteurs d'induction triphasés.

La Compagnie Westinghouse a déjả équipé un grand nombre de lignes avec le système monophasé. La première installation mise on service a été un tronçon de la voie ferrée d'Indianapolis à Cincinnati, dont la longueur totale atteindra 190 kilomètres, et sur laquelle la traction électrique doit se substituer complètement à celle à vapeur. Une station centrale produit du courant biphasé à 33000 volts. Des transformateurs monophasés, installés dans des sousstations distantes les unes des autres de 16 à 18 kilomètres, abaissent la tension ả 3300 volts. La ligne aérienne est à suspension catenaire, le fil de trolley étant suspendu tous les 3 mètres à un câble en acier qui est fixé à de solides isolateurs, portés par les consoles des poteaux.

La ligne interurbaine d'Indianapolis à Cincinnati est reliée aux réseaux de tramways de ces deux villes, exploités au moyen de courant continu a 500 volts. En outre, a la traversée des villes intermédiaires, la tension du courant monophasé est abaissée à 500 volts, de sorte que les automotrices doivent pouvoir fonctionner indifféremment soit sur des courants monophasés à 3000 ou 500 volts, soit sur du courant continu à 500 volts.

Chaque automotrice est munie de 4 moteurs séric, de 75 HP. Chacun d'eux comporte un induit analogue à un induit ordinaire à courant continu, mais pourvu de connexions équipotentielles, et muni de jonctions résistantes entre les bobines induites et le collecteur. L'inductcur est constitué par des anneaux en tole, soutenus par une carcasse en acier coulé ; ces anneaux portent des saillies en forme de pôles dans lesquels sont percées des encoches Inngitudinales mi-fermées. Chaque pôle a deux enroulements parcourus en série par le courant total : un enroulement inducteur, qui embrasse tout le noyau magnétique, et un enroulement compensateur, réparli dans les encoches, et destiné à neutraliser la réaction d'induit.

Pour la marche en alternatif, un auto-transformateur, dont le primaire porte deux prises de courant correspondant à 300 et 500 volts, abaisse la tension à deux valeurs secondaires différentes. Les moteurs sont loujours en parallèle, et les vitesses intermédiaires sout oblenues par insertion de résistance dans le circuit.

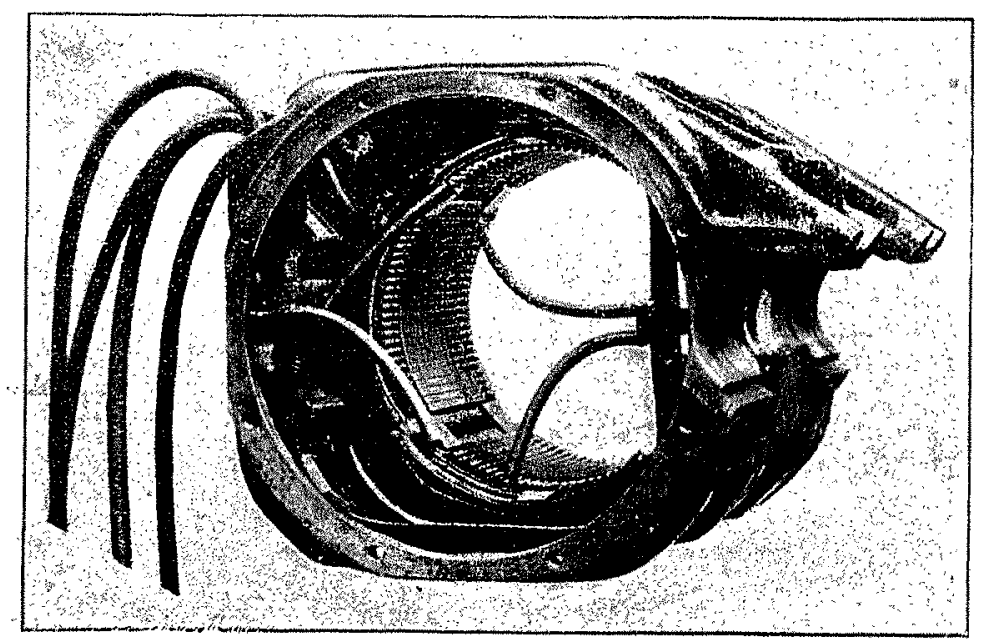

Fia. 12. - Vue de linducteur d'un moteur série Weslinghonse.

La même Compagnie a équipé la ligne d'Allanta a Marietta, longue de 24 kilométres, qui est exploitée au moyen de courant monophasé à 2200 volts en rase campagne et à 500 volts dans les villes. L'énergie électrique est produite sous forme de courants triphasés a 22000 volts, 25 périodes; la ligne est divisée en trois sections alimentées chacune par l'une des phases du systeme triphase.

Chaque automotrice porte 4. moteurs de $50 \mathrm{HP}$, groupes deux par deux d'une façon permanente. Un auto-transformateur abaisse la tension, et porte six prises de courant correspondant à six différences de potentiel graduellement comprises entre 144 et 228 volts. On peut ainsi faire varier progressivement la différence de potentiel aux borues des moteurs, sans perdre de l'énergie dans les résistances. Pour éviter, lorsqu'on passe d'une touche á la suivante, de mettre en court-circuit une section du transformateur, on emploie un dispositif analogue á celui des réducteurs de 
batteries d'accumulateurs. Ce dispositif consiste essentielment en une bobine de self et une résistance, qui sont toujours à cheval sur deux touches voisines, et au milieu desquelles est dérivé le conducteur allant aux moteurs.

Parmi les nombreusies autres lignes équipées en monophasé par la Compagnie Westinghouse, nous pouvons citer celle du chemin de fer électrique de Spokane and Inland qui, après achèvement complet, sera longue de 170 kilomètres. L'énergie électrique est produite suus forme de courants triphasés à 4000 volts 60 périodes, et transmise à une sous-station, située à 16 kilomètres de Spokane, qui transforme le courant triphasé en courant monophasé à 2200 volts, 25 périodes, au moyen de quatre groupes composés chacun d'un moteur asynchrone triphasé, action- locomotives de grande puissance dont l'une, de $1500 \mathrm{HP}$, a six essieux, attaqués chacun par un moteur do $250 \mathrm{HP}$, et assure le service des ateliers de la Compagnie à Pittsburgh.

Elle a construit aussi les locomotives de la ligne de New: York, New-Haven et Hartford, qui ont 4 essieux, portant chacun un moteur de $250 \mathrm{HP}$ à ventilation artificielle. enfilé sur lui, et l'entraînant par accouplement élastique. Deux essieux forment un bogie, et ils sont électriquement reliés en sêrie, formant un groupe qui absorbe 450 volts en alternatif, et 600 volts en continu. Le courant est continu à New-York, et alternatif à 11000 volts en rase campagne, ce dernier est abaissé dans des transformateurs statiques dont les secondaires portent six prises de courant, pour les différentes vitesses.

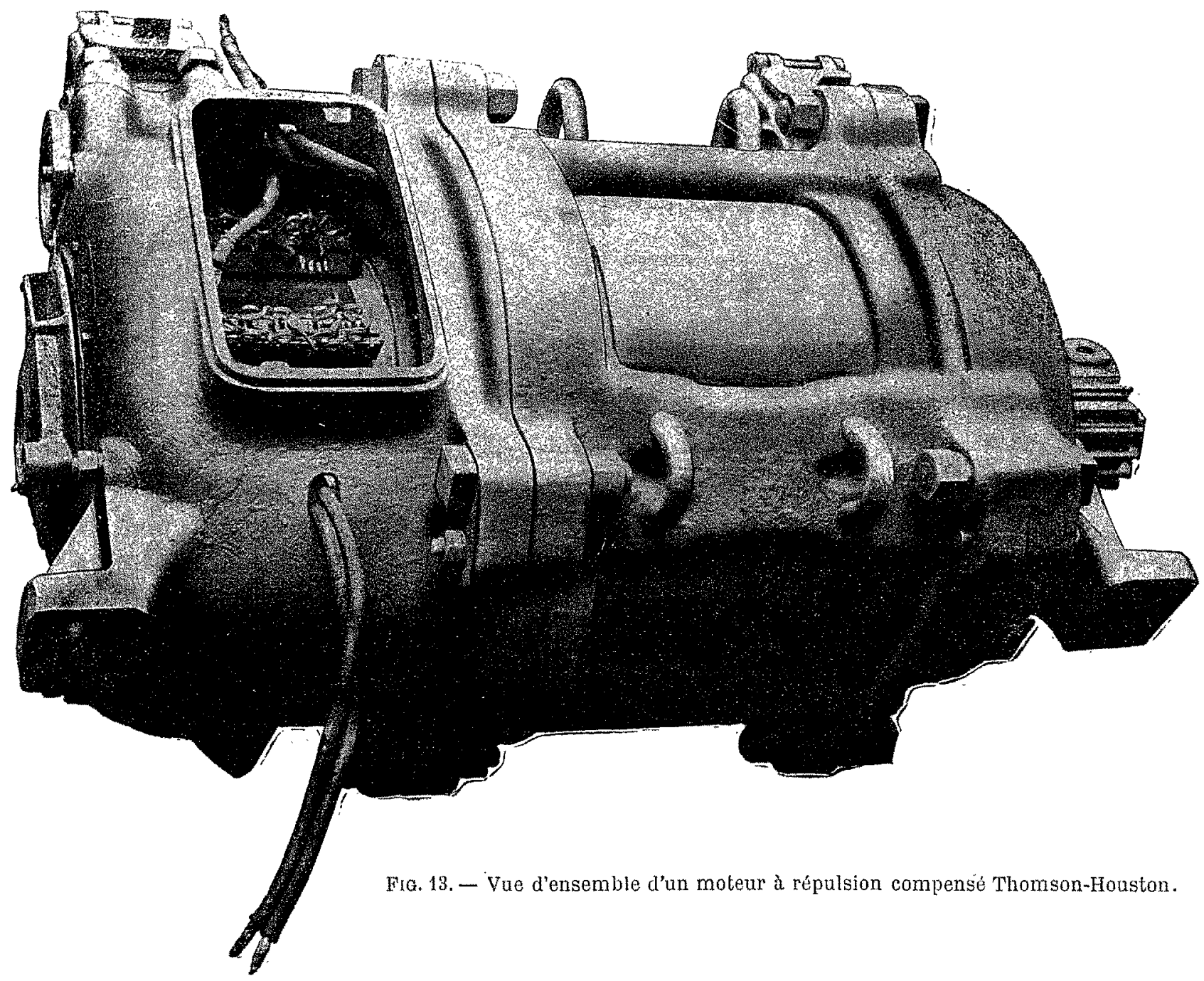

nant un alternateur monophasé et une dynamo à courant continu reliée à une batterie tampon. Des transformateurs élèvent à 45000 volts la tension du courant monophasé, qui est transmis à 15 postes de transformateurs statiques, répartis le long de la voie, et abaissant la tension à 6600 volts en rase campagne et à 700 volts dans les villages. Dans les villes, la ligne est alimentée par courant continu à 600 volts.

Le fil de trolley est à suspension catenaire. Les locomotives sont munies de 4 moteurs de $150 \mathrm{HP}$; les automotrices sont munies de 4 moteurs de $100 \mathrm{HP}$. Le réglage de la vitesse est effectué par variation de la tension aux bornes des moteurs, qui sont connectés en série d'une facon permanente.

La Compagnie Westinghouse a construit aussi quelques
Notons encore le tronçon de 6 kilomètres de la ligne de Port-Huron (Michigan) à Sarnia (Ontario) qui passe en tunnel sous le lit du fleuve. Les locomotives sont composées de deux unités accouplées, possédant chacune trois essieux attaqués par trois moteurs de $250 \mathrm{HP}$. Ces locomotives pèsent 125 tonnes et peuvent développer aux crochets un effort de10 tonnes pour une vitesse de $16 \mathrm{kms}$ à l'heure.

En Europe, le chemin de fer à voie étroite de Rome à Civita Castellana (74 kilomètres) a été équipé en monophasé par la Compagnie Westinghouse. Le courant de ligne est de 6000 volts en rase campagne et de 550 dans le voisinage de Rome.

La General Electric $C^{\circ}$ a fait aussi quelques applications du moteur série monophasé. Une première ligne a été 
équipée entre Schenectady et Ballston, et est alimentée à 2200 volts et 25 périodes en dehors des villes, et en 600 volts dans les villes. Chaque automotrice est munie de 4 moteurs de 50 HP, bobinés pour 200 volts, el groupés par deux en série d'une façon permanente; la variation de la vitesse s'obtient par un réglage série parallèle des deux groupes moteurs.

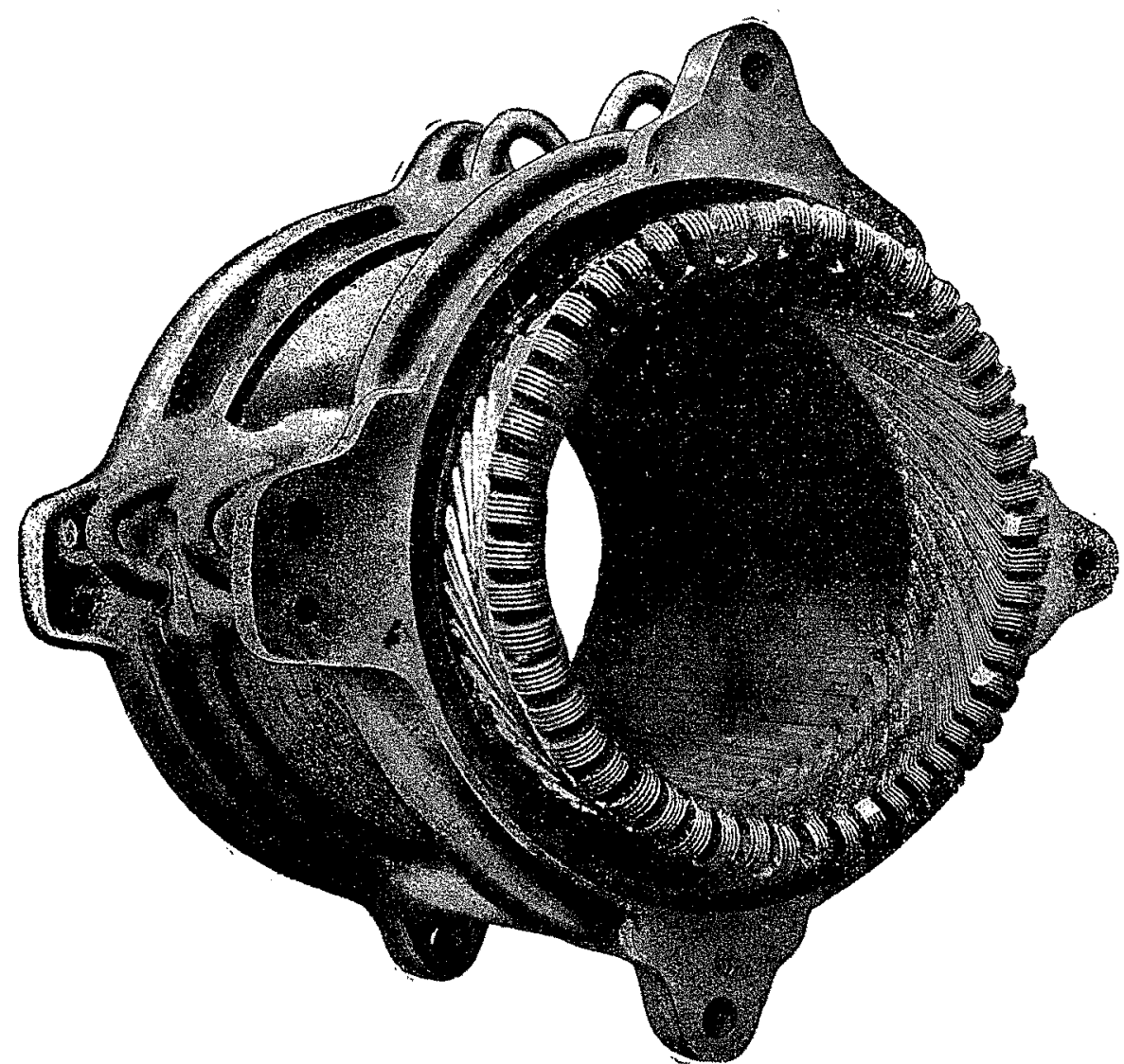

FIG. 14. - Vue de l'inducteur d'un moteur à répulsion compense Thomson-Houston,
La Compagnie francaise Thomson-Houston a appliqué en France le moteur à répulsion compensé au tramrvay du square Malakoff et du Clos Montholon aux Halles, a Paris. Le fil de trolley est a la tension de 550 volts; les essieux sont attaqués par deux moteurs Gratzmuller de 50 HP, à 300 volts; 25 périodes. Le réglage de la vilesse est effectué par la variation du voltage aux bornes des moteurs, au moyen d'un transformateur à plusieurs bornes secondaires.

L'Allgemeine Elektricitaets Gesellschaft a équipé en Belgique le réseau des chemins de fer vicinaux à voie étroite du Borinage, dont la longueur totale atteindra 129 kilometres. L'amenée et le retour du courant (monophase a 600 volts, 40 périodes) se font par fil aérien. Chaque automotrice, porte deux moteurs Winter-Eichberg à répulsion compensé, à six pôles, et d'une puissance de 40 chevaux. Cette Société a également équipé la ligne de Hambourg à Altona qui n'est que partiellement achevée $\left(^{*}\right)$.

Les Aleliers dOerlikon ont construit une locomotive, établie pour être alimentée sous 15000 volts, reposant sur deux bogies. Chacun de ceuxci porte, entre ses deux essieux, un moteur de $200 \mathrm{HP}$ comprenant un induit ordinaire à courant continu et un inducteur en deux pièces, portant huit pôles saillant et formés de tôles découpées. Chaque pôle est muni d'un enroulement compensateur, réparti dans deux encoches, et parcouru par le courant principal; il y a, en outre, huit pôles de commutation, dont les bobines sont parcourues par un courant, de phase et d'intensité telles qu'il induise, dans les bobines en court circuit, une force électromotrice capable de neutraliser les forces électromotrices

Les expériences faites ont montré que, pour la traversée des villes en basse tension, il était préférable d'employer du courant continu, car, à la fréquence 25 , la résistance apparente des rails était égale à 6,5 fois la résistance ohmique. nuisibies a une bonne commutation.

La Société Siémens-Schuckert a équipé la ligne de Murnau à Ober-Ammergau (23 kilomètres), dont le profil est

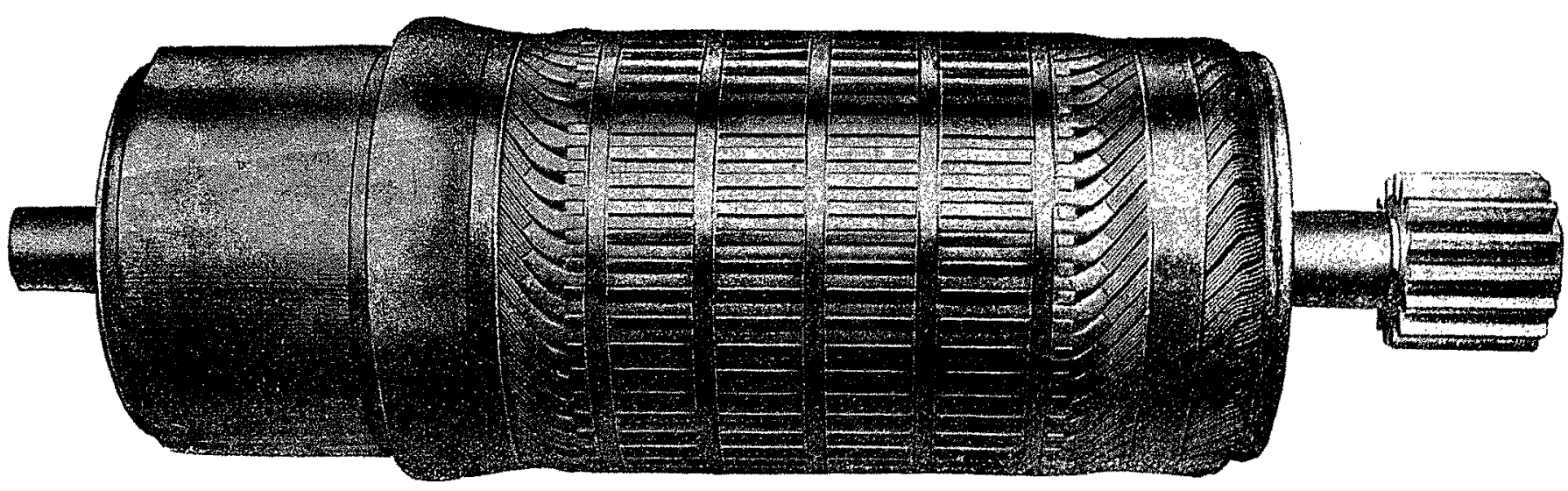

Fig. 15. - Vue de l'induit d'un moteur à répulsion compensé 'Thomson-Houston.

Une seconde ligne est celle de Bloomigton, Pontiac and Joliet, dont la longueur totale atteindra 144 kilomètres. Elle est alimentée sous 3300 volts, 25 périodes. Chaque automotrice est munie de 4 moteurs de $75 \mathrm{HP}$, bobinés pour 200 volts, et en série d'une façon permanente. Un transformateur abaisse la tension, et porte cinc prises de courant faisant varier la tension de 400 à 800 volts: très accidenté, avec du courant monophasé à 5000 volls, 16,66 périodes. Les automotrices ont trois essieux, dont chacun est attaqué par un moteur de $100 \mathrm{HP}, 250$ volts, qui est aussi muni de pôles de commutation.

(*) Voir La Houille Blanche de novembre 1900 fChemin de fer úlectrique Blankenese-Ohisdorf). 
Remarques. - En général, la transmission des moteur's aux essieux se fait par engrenages. Ce système est très avantageux, car il rend indépendantes les vitesses angulaires de ces deux engins, et permet, tout en adoptant pour un type de moteur déterminé la vitesse de rotation la plus avantageuse, de faire varier la vitesse de l'essieu dans des limites assez étendues, suivant les applications envisagées.

Mais, pour les locomotives à grande puissance et à grande vitesse, il y a intérêt à supprimer les organes réducteurs de vitesse. Ainsi, si l'on considère une locomotive faisant $100 \mathrm{kms}$ à l'heure. et ayant des roues de $1 \mathrm{~m} 10$ à $1 \mathrm{~m} 35$, celles-ci tourneront à raison de 4 à 500 tour par minute, ce qui convient dune manière parfaite à des moteurs de 3 à 500 HP. En pratique; la suppression des organes réducteurs de vitesse devient logique toutes les fois qu'il s'agit de moteurs de plus de $250 \mathrm{FP}$, et de vitesses de marche supérieures à 80 kms à l'heure.

Le poids non suspendu, reposant sur l'essieu moteur, doit rester limité au dessous de la valeur à partir de laquelle les effets d'inertie auraient une influence fàcheuse sur la bonne conservation des voies. On est donc conduit à rejeter les combinaisons daccouplement direct, pour lesquelles le poids entier du moteur est porté par l'essieu. Cette dispo- même circonférence, et décalés de $60^{\circ}$. Ce dispositif a l'avantage de faire démarrer la moteur avant le train, de sorte que, au moment du départ, la force contre-électromotrice a déja une certaine valeur, ce qui diminue les surcharges brusques dans les stations.

M. Mazen, étudiant la locomotive du New-York Central, montre que ce typë ne parait pas avantageux. Les entrefers devant rester constamment égrux, si l'on ne veut pas s'exposer à des attractions inégales de pôles, lesquatre essieux doivent toujours rester rigoureusement parallèles. D'autre part, lorsque le courant est lancé dans les moteurs, les induits sont plongés dans un champ magnétique dont l'action a pour effet de s'opposer aux déplacements verticaux. II en résulte une certaine raideur des organes de roulement, ce qui permettrait peut-être d'expliquer le récent déraillement survenu dans une courbe de la banlieue de Netw-York.

M. de Traz fait remarquer qu'il importe de ne pas trop réduire le nombre des combinalsons au démarrage. Les accélérations adoptées sont, en effet, de plus en plus fortes. Or, ce n'est pas la valeur élevée de l'accélération qui peut incommoder le voyageur, c'est la variation brusque de cette accélération qui se produit à chaque passage d'une combinaison à la suivante.

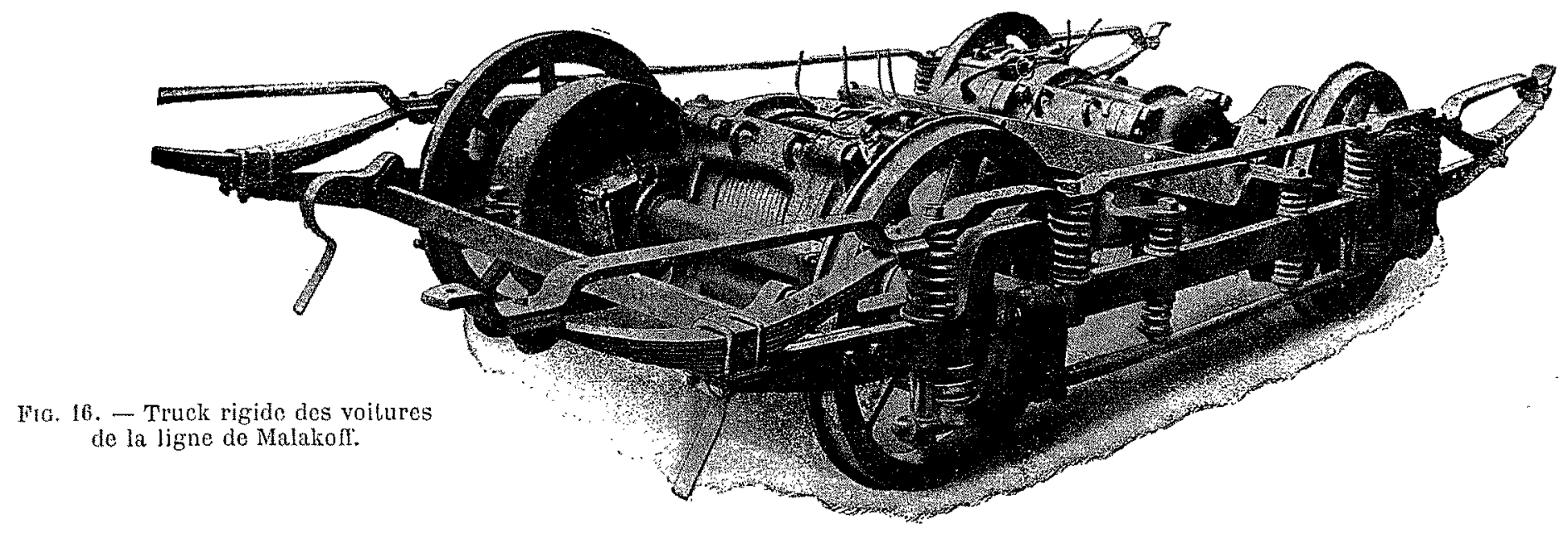

sition, essayée sur les locomotives du Central London, a eu un insuccès complet, pour des motifs d'ordre purement mécanique.

On a ainsi été conduit à l'accouplement direct sur un arbre creux, concentrique à l'essieu et accouplé élastiquement avec lui, notamment sur les locomotives Heilmann, sur celles du Ballimore and Ohio, du New-York, NewHaven and Hartfort, de Ia ligne Paris-Versailles, sur les locomotives d'essai de Zossen.

Dans d'autres combinaisons, le rotor est directement calé sur l'essieu lui-même, dont il fait partie intégrante, le stator élant fixé au châssis et possédant, par rapport à l'essieu, une suspension élastique. Tels sontles dispositils employźs sur le Nezo-York Central, et celui imaginé par M. Auvert.

Les moteurs des locomotives électriques de la ligne de, Paris à Versailles sont montés sur des tubes concentriques à l'essieu. Le diamètre maximum du corps de l'essieu est $170 \mathrm{~mm}$. le jeu entre le corps et le tube est de $50 \mathrm{~mm}$. A la vitesse maxima de $100 \mathrm{kms}$ à l'heure, el avec des roues de $1^{\mathrm{m}} 3 \mathrm{~L}$, la vitesse tangentielle dans les coussinets est de $6^{\mathrm{m}} 70$ à.la seconde, malgré cela, le système donne d'excellents résultats. La liaison de l'essieu au tube creux se fait au moyen de six ressorts à boudins réunissant entre eux trois points du plateau et trois points de la roue, placés sur une

\section{LA HOUILLE BLANCHE ET LA QUESTION SYLVO-PASTORALE}

Conférence faite aux élèves de l'Institut Electrotechnique de Grenoble, par notre collaborateur, le Commandant Audebrand, sous les auspices de la Société pour le développement de l'Enseignement technique près l'Université de Gronoble.

\section{Messieurs,}

C'est pan application du dernier partagraphe de l'art. 4 de ses staluts que la Société pour le développement de l'ensoignement technique, qui patronne votre Institut, mia demandé de venir aujourd'lui vous endretenir des questions sylvo-pastorales dans leurs rapporls avec l'hydraulique.

C'est là, en effet, une question d'une très haute aclualité sur laquelle les ingénieur's dorvent être édifiés, et à laquelle ils doivent apporter' une attention soutenue.

Cependant cel ne sont pas là malières d'examen, en ce sens qu'elles ne sont pas axigées. pour l'obtention du diplóme dont vous poursuivez la possession, et, de ce point de vue étroit ef restreint, vous pourriez pout-être vous croire aitorisés à vous désintéresser de leur étude. C6 serait à tort : il n'e faut pas songer uniquemenl à l'examen de fin d'études, mais encore à cet examen bien autrement complexe et redoutable qu'est la vie. Si vos maitres ont le devoir de vous conduire à lobtention du diplôme d'ingénicur, ils ont aussi le devoir non moins impérieux de vous armer 